\section{Aplicación de Moodle para fortalecer el proceso de enseñanza de inglés en la educación abierta}

\author{
Application of Moodle to strengthen the process of teaching \\ English in open education
}

\section{Chriselle Domenica Espinar *}

\section{RESUMEN}

Introducción. La enseñanza a través de la plataforma Moodle en la educación en línea, se entiende como aquella que solicita un desarrollo autónomo asistido u orientado por uno o más docentes en la educación superior. En la actualidad existe la necesidad de aprovechar Moodle para fortalecer la enseñanza de inglés. Objetivo: Este artículo analiza las diferentes producciones científicas orientadas a la plataforma Moodle y sus beneficios, considerando sus inconvenientes, para fortalecer el proceso de enseñanza en inglés e intenta proponer alternativas metodológicas en la utilización de Moodle. Materiales y métodos. Esta revisión de $5 \mathrm{I}$ estudios es de alcance descriptivo cualitativo, se extrajo; recomendaciones, resultados, metodologías al aplicar Moodle y se organizó en tres apartados; (I) Beneficios de aplicar Moodle en la enseñanza de inglés, (2) Inconvenientes que pueden presentarse al aplicar Moodle en la enseñanza de inglés, (3) Actividades en Moodle y alternativas metodológicas para las clases de inglés en línea. Resultados. Se descubrió que las habilidades comunicativas más desarrolladas al integrar Moodle tenían en común la mejora en la gramática y escritura. Conclusión: Se puede tomar a este artículo de referencia para revisar todos las características e inconvenientes que ofrece Moodle y fortalecer la enseñanza del inglés en línea.

\footnotetext{
*Estudiante de tercer semestre en Pedagogía de los Idiomas Nacionales y Extranjeros, Universidad de Guayaquil, correo electrónico institucional: chriselle.espinarc@ug.edu.ec , correo electrónico personal: chriselledomenica@hotmail.com, ORCID: https://orcid.org/0000-0003-1273-8I IX, Google académico: Chriselle Domenica Espinar
}

\section{REVISTATECNOLÓGICA ciencia y educación Edwards Deming}

ISSN: 2600-5867

Atribución/Reconocimiento-NoCo mercial- Compartirlgual 4.0 Licencia Pública Internacional — CC

\section{BY-NC-SA 4.0}

https://creativecommons.org/licenses /by-nc- sa/4.0/legalcode.es

Editado por: Tecnológico Superior Corporativo Edwards Deming

Enero - Julio Vol. 5 - I - 202 I

https://revista-edwardsdeming.com/index.php/es e-ISSN: 2576-097I

Recibido: 04 Marzo 2020

Aprobado: II Diciembre, 2020

Pag 8I-97 
Palabras clave: Integración de Moodle; modalidad en línea;

Proceso de enseñanza-aprendizaje; Idioma inglés

\section{ABSTRACT}

The teaching process via the Moodle platform in online education is understood as one that demands autonomous development assisted or oriented by one or more tutors in higher education. Currently, there is a need to take advantage of Moodle to strengthen English teaching. Objective: This article discusses the different scientific productions oriented towards the Moodle platform and its benefits, considering its weaknesses to strengthen the teaching process in English, and tries to propose methodological alternatives in the use of Moodle. Materials and methods. This review of $5 \mathrm{I}$ studies ranges from qualitative to descriptive fields; extracted: recommendations, results, and methodologies when applying Moodle-- organized into three sections; (I) Benefits of applying Moodle in English teaching, (2) Inconveniences that may occur when applying Moodle in English teaching, (3) Moodle Activities and methodological alternatives for online English teaching. Results. The study recognized that the most developed communicative skills when integrating Moodle had in common the improvement in grammar and writing. Conclusion: This article can serve as a reference to review all the qualities and disadvantages offered by Moodle and strengthen the teaching of English online.

Keywords: Moodle integration; online modality; Teaching-learning process; English language

\section{INTRODUCCIÓN}

A lo largo del tiempo se han ideado muchas formas para la enseñanza de un idioma que en la actualidad ya no son tan efectivos, tanto la comunicación y la forma de transmitir un mensaje es diferente de lo que se conocía antes y seguirá cambiando progresivamente con la aplicación de las nuevas tecnologías en la educación. En ese sentido la Agenda Educativa Digital 2017-202I informa que; a comienzos del año 2000 en América Latina se empieza a diseñar una política pública integral de sociedad de la información, con el propósito de crear e implementar programas para el acceso y uso de las TIC (Ministerio de Educación del Ecuador, 2017). Con la finalidad de lograr que la enseñanza se transforme en un proceso estimulante en las diferentes áreas en las que el individuo se desenvuelva (García \& López, 20l4).

Esto hace que actualmente a nivel educativo los docentes usen nuevas formas de enseñanza adaptándose a estas herramientas tecnológicas de acceso a la información y gozando de sus grandes beneficios en todas partes y en cualquier momento (Granados et al., 2019). Por ello Mosquera et al. (2019) subrayan que “ [...] la didáctica a emplearse debe partir de un enfoque innovador de formación pedagógica/andragógica, que trate un planteamiento didáctico de cómo utilizar la tecnología en clase. " (p.93). Es por esta razón que los docentes se enfrentan a una gran tarea al implementar estas nuevas tecnologías en la educación en línea, así como también el de desarrollar habilidades 
técnicas y didácticas. A su vez Figueroa et al. (2019) plantea que ahora unos de los más importantes desafíos en el ámbito educativo es el de replantarlo y acomodarlo a los nuevos cambios que enfrenta la sociedad contemporánea.

Según los autores Gómez Domínguez et al. (2019) particularmente el campo de enseñanza en lenguas extranjeras se ha manifestado como " [...] abierto y flexible a las innovaciones y a la incorporación de nuevas herramientas para el apoyo de los procesos de enseñanza y aprendizaje." (p.77). Lo que demanda hoy en día a emplear plataformas educativas para potenciar el uso de las tecnologías de la información y la comunicación en la enseñanza de inglés.

Desde la perspectiva de Macancela et al. (2019) los ambientes virtuales son una buena opción ya que dan paso a la oportunidad de interactuar de diferentes maneras, es decir de forma sincrónica o asincrónica, todo esto se complementa para la correcta construcción del conocimiento. Se entiende así que las plataformas e-learning como Moodle abren estos espacios activos de comunicación y aprendizaje.

Arroyave et al. (2020) destaca también que estos modelos de aprendizaje en línea dan una nueva percepción de los modelos de enseñanza en la educación superior, en donde ahora se coloca al estudiante en el centro del proceso, lo que demanda una actualización de metodologías, objetivos, las formas de transmitir los saberes, entre otros.

Pese a que se menciona que ya existen numerosos estudios que han tratado la utilización de las TIC especialmente en la enseñanza universitaria a escala nacional como internacional precisando el uso de Moodle (Cole y Foster, 2007; Rodríguez et al., 2009; Pérez et al., 2009 citado en Santamaría et al., 20I2). Aun se presenta la necesidad de saber aprovechar Moodle para fortalecer la enseñanza del idioma inglés. De esta forma y de acuerdo con (Domínguez et al., 2019) permanece deficiente el conocer con exactitud para que se emplea este tipo de herramienta educativa en la enseñanza del inglés.

Por otro lado, el contexto se ubica en Guayaquil-Ecuador que por circunstancias de emergencia sanitaria tomadas en respuesta al COVID-19 la Universidad de Guayaquil (2020) anunció que sus actividades académicas formativas se realizaran bajo la modalidad en línea por diferentes plataformas, específicamente Moodle, Zoom y Teams pretendiendo constar la interacción sincrónica y asincrónica.

Se plantea de ese modo la enseñanza de inglés a treves de la plataforma Moodle en la educación abierta en línea que toma lugar en la educación superior a nivel local, donde educación abierta si bien conforme a lo dicho por Zawacki-richter et al. (2020) se conceptualiza como un grupo de prácticas educativas el cual la noción de acceso libre tiene muchas connotaciones y su concepción se ha expandido todavía más con el tiempo. Adicionalmente Lane (20I3) menciona que la educación abierta por su parte tiene un valor significativo al momento de crear oportunidades y experiencias compartiendo libremente el aprendizaje y la enseñanza.

Sin embargo, desde la postura de este articulo la definición operacional de educación abierta se entenderá como aquella que solicita un desarrollo autónomo asistido u 
orientado por uno o más docentes, pudiendo ser virtual o en línea, tomando esta última modalidad en concreto para la visión de este apartado.

Considerando el conocimiento insuficiente que se tiene para aprovechar la implementación de Moodle en el proceso de enseñanza de inglés en este contexto, artículo de revisión tiene como objetivo analizar las diferentes producciones científicas orientadas a la aplicación de esta plataforma e-learning y sus beneficios, tomando en cuenta también los inconvenientes que se pueden presentar al momento de aplicar Moodle, con el propósito de fortalecer así el proceso de enseñanza del idioma inglés e intenta proponer alternativas metodológicas en la correcta y equilibrada utilización de Moodle.

\section{MATERIALES Y MÉTODOS}

Se realizó un diseño de revisión bibliográfica de alcance descriptivo cualitativo de documentos de producción científica dirigido a la enseñanza del idioma inglés de igual manera estudios de casos y estudios científicos.

Como primer paso se efectuó una búsqueda en las siguientes bases de datos; Eric, Redalyc, Scielo y REDIB de documentación en educación por diferentes instituciones y entidades profesionales tanto en Ecuador como en el contexto internacional sobre Moodle con respecto al fortalecimiento en la enseñanza de inglés.

Se considero apropiado realizar la búsqueda con las palabras claves en español y principalmente en inglés porque en este idioma se encontraron más estudios publicados que en español. Más adelante se realizó la recopilación de estudios de casos, revisiones documentales y estudios científicos a través de la siguiente ecuación principal de búsqueda; "Moodle" AND "English teaching processes" y en español "Moodle" y "enseñanza en ingles" sin límite de fecha, la cual posteriormente se modificó en pequeñas proporciones para la búsqueda de resultados significativos.

Se tomaron en cuenta también las referencias bibliográficas de algunos de los artículos más relevantes con la finalidad de recuperar otros estudios sustanciales al tema de revisión. Estos artículos fueron hallados por medio de Redalyc, y de DOAJ.

Cuando se realizó la búsqueda de información se incluyó todos aquellos documentos que contuvieran aportes de diferentes disciplinas educativas y que a su vez correspondieran a la utilización de Moodle como parte de un proceso de enseñanza y un entorno de aprendizaje.

En cuanto a los estudios de caso y estudios científico se empleó como criterio de inclusión que estos estudios sean aplicados principalmente a nivel universitario, de igual manera que incluyeran la enseñanza en el idioma inglés. Asimismo, se incluyeron aquellos estudios que añadieran en sus conclusiones sugerencias de la aplicación de Moodle en clases en línea al igual que clases semipresenciales.

El principal criterio de exclusión fue que en los artículos no encerraran información respecto a las TICS como herramienta para la enseñanza y un entorno de aprendizaje en clases en línea o semipresenciales. 
Posterior a la búsqueda Inicial se encontraron 263 estudios en los cuales más tarde se excluyeron 212 que no aportaban resultados significativos a esta revisión. Se seleccionaron 4 estudios de casos, 2 estudios originales, I ensayo y 44 artículos.

Para la extracción y selección se verificó los respectivos resúmenes de cada documento, además, en ciertos casos fue necesario la lectura completa de los artículos con el objetivo de comprobar que la información se relacionara o no con la finalidad de esta revisión y eventualmente recuperarla o descartarla, con respecto a la organización de las referencias se utilizó el gestor bibliográfico Mendeley.

Del conjunto de $5 \mathrm{I}$ estudios revisados se extrajo la información más relevante, conteniendo recomendaciones, resultados, metodologías al aplicar Moodle para la enseñanza del idioma inglés en línea, el cual se organizó en tres apartados; el primero dirigido a los artículos que hablan de los beneficios de aplicar Moodle en la enseñanza del idioma inglés, el segundo; aquellos que indican inconvenientes que pueden presentarse al aplicar Moodle en la enseñanza de Inglés, el tercero; actividades en Moodle y alternativas metodológicas para las clases de inglés en línea. Además, de todos los estudios se extrajo autores, año, fuentes de información, revistas en la que fueron publicadas, sus respectivas conclusiones y país donde tuvo lugar el estudio, para poder identificar cual tiene más publicaciones respecto al tema.

\section{RESULTADOS}

En primera estancia Zhou et al. (2020) indica que se utilizan todas aquellas ventajas que tiene las tecnologías de la educación actual para que en las clases online se pueda crear un ambiente interactivo y de calidad. Resalta también que la enseñanza de manera online es un referente importante de futuros modelos de enseñanza (Zhou et al., 2020). Por lo cual el usar herramientas que den paso a la comunicación constante va a reflejar un efecto positivo en la experiencia de los estudiantes y en su compromiso, así lo muestra Park (2020) en su estudio de experiencias de las clases en línea. Además la educación de manera en línea requiere que los estudiantes tome una postura de responsabilidad por su propio aprendizaje según Knowles (2007).

Gracias al sistema que manejan las plataformas e-learning como Moodle, estas pueden acumular grandes cantidades de información y ayuda al profesor a tener un mayor control de la participación del estudiante dentro del curso, a su vez esta información es analizada y revisada por el mismo (Zhang et al., 2020). En este sentido el docente podrá identificar las posible dudas o preguntas que se susciten por parte de los estudiantes, así mismo como colocar información que ayude a responder esas inquietudes. De forma se entiende que ha dado una oportunidad a los docentes el emplear estas herramientas para fortalecer la calidad de enseñanza y aprendizaje en el idioma inglés (Gunduz \& Ozcan, 2017).

El estudio de Pérez et al. (2010) afirma así que estas plataformas toman un importante valor pedagógico cuando se utilizan como herramientas facilitadoras en la comunicación entre el docente y el estudiante en la construcción del conocimiento. Esta comunicación es posible por medio de los foros o el chat que incluye la plataforma, así mismo en la 
página principal se puede agregar ciertas actividades y visualizar aquellas que están pendientes (Espinoza et al., 2019). De tal manera Susanti (2008) en su investigación sugiere que durante el proceso de enseñanza en línea, los docentes deben abrir la puerta a oportunidades en donde el estudiante comunique sus ideas. Es decir, un espacio creado por el maestro que apoye a la participación de manera activa a través de Moodle.

Por otro lado el estudio realizado por Seluakumaran et al. (2020) señala que Moodle se presenta como una plataforma amigable con varias opciones interactivas que pueden potenciar el aprendizaje de los estudiantes y da mayor flexibilidad al proceso de enseñanza. En consecuencia Yafaei \& Attamimi (2019) concluyen que Moodle como plataforma es una herramienta que evidentemente debe usarse dentro de la enseñanza del idioma inglés.

En esta línea también mencionan que Moodle establece una gran relevancia a nivel universitario (Yafaei \& Attamimi, 2019). Por consiguiente, Rey et al. (2009) precisan que Moodle se posiciona en un lugar destacado en comparación a otras plataformas elearning debido a que es inclusiva y flexible al ser una plataforma de código abierto. Pese a que Alcántar et al. (20l8) revela en su estudio que estas plataformas se aprecian como positivas en la adquisición de un segundo idioma, identifica que aún hay que interiorizar estas en el proceso formativo.

Otros estudios Mostraron que al momento de aplicar Moodle en la enseñanza en línea combinado con los materiales expuestos en la plataforma, los estudiantes tuvieron más confianza al expresar sus ideas, sintiendo menos miedo ya que no tuvieron que exponerse frente a otros compañeros del curso (Díaz., 2012; Nagi \& Bojiah, 2020). Sin embargo, aunque esto si favoreció a que puedan desenvolver sus ideas abiertamente Nagi \& Bojiah (2020) recalcan que esos resultados obtenidos son de gran ayuda y esenciales solo si se cuenta con el soporte y material adecuado del docente independientemente si las clases son en línea o no.

Desde el punto de vista didáctico como describe Yamisleydis Pérez Peña, Victor Enmanuel Lorenzo Pérez (2020) Moodle admite agregar diferentes textos, imágenes incluso audios que permiten desarrollar las diferentes habilidades comunicativas del idioma inglés. Por esta razón en el (2019), Nur en los resultados de su investigación menciona que casi todos los estudiantes percibieron tanto la enseñanza como el aprendizaje de la gramática en ingles utilizando Moodle fue interesante y eficiente. Asimismo estos resultados también concuerdan con Vega-carrero et al. (2017) donde encontraron que a través del aula virtual se logra maximizar el vocabulario y habilidades en gramática como en lectura de forma notoria. Aunque cuando se trata de compresión lectora Hsiao-fang (2015) recomienda contar con el apoyo del docente además del material así pues esta habilidad incrementa aún más.

Desde otra perspectiva Alcántar et al. (20/8) en su estudio hace hincapié que los docente al usar estas plataformas para subir actividades e información lo convierten en un espacio donde almacenan datos, a su vez esto desanima e impone limitaciones. Esto puede convertirse en un problema en el proceso de enseñanza, ya que genera 
desmotivación en los estudiantes y no se aprovecharía las funciones pedagógicas que esta plataforma puede brindar.

Esta perspectiva igualmente corresponde a las conclusiones de Peralta et al. (20l I) sosteniendo que la plataforma Moodle en gran parte es usada por los estudiantes para descargar archivos que anteriormente fueron publicados por los docentes, así pues los foros y el chat son muy limitados en su uso. Se entiende que lo aplican de forma administrativa para la entrega o designación de tareas. Esto se consta en otros resultados en los cuales arrojaron que los docentes tienen un dominio didáctico insuficiente de Moodle, lo que conlleva a que se tome esta plataforma para la entrega de contenido, provocando que los estudiantes sean guiados de forma pasiva y lo traslada a un enfoque conductista (Arteaga López, 20I I; Cabero-almenara et al., 2019).

Así pues desde otro orden de ideas, en la publicación de González y Medina (2012) desde la perspectiva de los estudiantes la cantidad de ejercicios para practicar lectura en ingles era agotador. Adicionalmente varios de ellos consultaron si se les podía dar más tiempo para poder finalizar esas actividades (González \& Medina, 20I2). De este modo el estudio de Canals y Robbins (2017) expone que los maestros deberían dar retroalimentación de las tareas, para que se realicen de forma efectiva y los estudiantes se comprometan más con la materia.

En cuanto a la plataforma Moodle Peña et al. (2020) resaltan que esta plataforma precisa ser renovada con nuevas opciones que promuevan aún más el aprendizaje de un nuevo idioma.

Con respecto al tiempo por parte de los docentes Bonfield et al. (2020) manifiesta en su análisis que durante el periodo de creación de un curso línea el tiempo es un factor que se vuelve considerablemente estrecho. Se puede deducir así que el proceso de planificación para esta modalidad se ve afectado significativamente al aspirar tenerlo preparado a tiempo y cumplir con todas las actividades asignadas.

Lamentablemente en el contexto de distanciamiento social que actualmente se debe cumplir, se ha convertido en un reto para los profesores el ajustar y sobrellevar las dificultades del aprendizaje en las clases en línea (Lutfiana \& Suwartono, 2020). Entre estas dificultades Gunduz y Ozcan (2017) nombra en su investigación que la mayor parte de los docentes mencionan que los estudiantes confrontaban inconvenientes con el acceso al sistema, problemas con las contraseñas, entre otros.

Conforme a esto, el estudio realizado por Srichanyachon (2013) indica que a causa de estos escenarios, es por lo que aún se prefiere estudiar ingles de manera presencial en las aulas de clase. Igualmente la falta de materiales, la poca capacitación en el uso de Moodle y como aplicarlo en ambientes e-learning se establecen como problemas principales (Jakshylykov \& Nurmatov, 2016).

Zhou et al. (2020) por su lado expresa que existe la necesidad en los docentes de reconstruir el currículo, para así mejorar las metodologías de enseñanza. Es por esta razón que en este apartado se menciona los estudios que dan recomendaciones para las actividades que se pueden realizar en la plataforma Moodle, así como también alternativas metodológicas a aplicar en las clases de inglés. 
Desde la posición del estudio realizado por Cabero-almenara et al. (2019) sostienen que cuando se habla de estas plataformas de educación virtual como Moodle, se trata de no visualizarlas como un espacio para recoger y guardar información, al contrario percibirlo como una herramienta que desarrolle el trabajo colaborativo y la creación en conjunto de los saberes. En otras palabras, se trata de aprovechar el potencial y los usos que ofrece esta plataforma a favor de la educación y considerar alternativas que den resultados exitosos.

Por ello y de acuerdo con Pérez et al. (2010) en su investigación exponen que la pieza primordial en conseguir la efectividad en estas plataformas esta en no concebirla de manera simplista, sino más bien diseñar un entorno interactivo donde el docente encamine y asista al educando, fomentando su creatividad y con dirección a cumplir los objetivos deseados.

Partiendo de este orden de ideas, con la gran ayuda de esta plataforma indudablemente si se cuenta con; los materiales precisos, la metodología ajustada a esta modalidad, el acceso a los dispositivos necesarios reconociendo sus pedagógicos, entre otros, se puede asegurar un impacto positivo en la efectividad al emplearlas, así lo señala en su estudios Aretio (20l8). Sin embargo, está implícito que los materiales a emplearse tienen que ser de buena calidad para que aporte así los resultados que se desea al momento de enseñar un segundo idioma.

En el caso de Moodle son los docentes quienes se encargarán de diseñar un entorno propicio para la enseñanza del idioma inglés en línea. Así pues Shaykina (2015) destaca que es necesario que los docentes tomen en consideración los posibles inconvenientes y sean flexibles ante estas situaciones. De esta manera algunas investigaciones manifiestan ciertas actividades que se pueden realizar en la plataforma.

Por ejemplo cuando se trata de las funciones de Moodle; Ghounane (2020) expone que el sistema de Moodle permite a los profesores programar actividades y diseñar pruebas a las cuales los estudiantes podrán acceder. Por otro lado en el 2017 Koneru enumera algunas funciones que permite Moodle entre las más principales; evaluaciones electrónicas, administración de la tareas, coordinación y entrega de contenido. De esta marea se agiliza y se gestiona de mejor forma el proceso de enseñanza en línea.

En esta misma línea y en correspondencia con la investigación de Seluakumaran et al. (2020) se pronuncian indicando que las funciones que permite Moodle dan paso a que los docentes organicen su contenido y el curso en general, otorgándoles la libertad de poder repartir el material necesario a la misma vez induciéndolos a utilizar métodos creativos de enseñanza. Asimismo Muhammad (2020) resalta que estas funciones incentiva a que los estudiantes opinen y posibilita dar retroalimentación ya todo el contenido está habilitado en la plataforma y puede ser visualizado por todo el curso.

Con respecto a las herramientas que Moodle ofrece para las actividades dentro de la plataforma en el 2018, Gómez menciona que Moodle permite elaborar ejercicios prácticos designando grupos pequeños invisibles, los cuales se puede ingresar y desarrollar el trabajo designado de forma simultánea en la hora asincrónica. 
De esta forma Luján-García y Garcia-Sánchez (20I5) adicionalmente subrayan que el foro de Moodle promueve el perfeccionamiento de las habilidades comunicativas en ingles concretamente en la escritura. En sus resultados mostraron también que los estudiantes que participaron en las discusiones en el foro y en conjunto con el glosario no solo mejoraron la escritura y su vocabulario considerablemente sino también el trabajo colaborativo (Luján-García \& Garcia-Sánchez, 20I5).

Por otra parte en los resultados de Torress-Cajas y Yépez-Oviedo (2018) indican que no solo se desarrolla y mejora la habilidad escrita, por su parte también se potencializa la oral, puesto que gracias al foro y los chats que proporciona el aula virtual los estudiantes compartieron sus ideas notablemente consiguiendo un entorno interactivo el cual se exige para aprender un segundo idioma.

En relación a la retroalimentación Joyce (2018) destaca lo beneficioso que es dar una respuesta continua a su desenvolvimiento. Así el alumno siente que está siendo guiado en las actividades designadas, desarrollando su confianza, motivándolo y disminuyendo el estrés que pude causar el aprender ingles a través de esta plataforma y en la modalidad en línea. No obstante Rey et al. (2009) subrayan que moderar la veces que como docente se interviene es primordial, si bien una retroalimentación constante en los foros puede verse como beneficio, esta puede ser desacertado, ya que establece una postura intimidante por parte del docente, asimismo no recomienda la demora de estas intervenciones, puesto que podría entenderse que el foro ha sido descuidado.

Debido a esto en los resultados de Ardila y Juan Rodrigo Bedoya (2006) los estudiantes que fueron entrevistados con respecto a la inclusión de Moodle en la modalidad en línea en un curso de gramática en ingles sugirieron; moderar la monto de actividades en la plataforma, que los profesores den retroalimentación de estas actividades, proporcionar más tiempo para el desarrollo de la mismas, fortalecer algunos los temas mas complicados, entre otros. En este orden González y Medina (2012) recalcan que en proporción a la a la cantidad de actividades designadas por el docente estas deben ser balanceadas.

Desde otro punto de vista y con la intención de crear alternativas para los docentes en la enseñanza del idioma inglés, se trae a consideración nuevamente lo mencionado por Luján-García y Garcia-Sánchez (2015) que mencionan lo necesario que es hablar desde una perspectiva y enfoque constructivista, activa y colaborativa con una enfoque primordial en la flexibilidad para la construcción del conocimiento en estos entornos. $\mathrm{Si}$ bien el constructivismo es ideal en estos espacios de enseñanza en línea no hay que descartar otros enfoques y metodologías.

Por ejemplo; Un estudio realizado en el 20II, Dávila rescata que "[...] no se puede negar a priori la aplicación de momentos conductistas sobre todo cuando no hay nada que descubrir y lo que se tiene que aprender ya está hecho y no hay lugar para nuevas interpretaciones" (p.I05). Se entiende así que se puede intervenir en ciertos momentos cuando hay que reforzar y corregir conceptos difíciles de comprender como lo puede llegar a ser en inglés; la gramática o escritura sin embargo esto no quiere decir que dentro del aula virtual se elogie o se sancione. 
Por lo que se necesita de una alternativa factible y de aplicación sostenible que además considere la utilidad y el potencial de emplear las funciones, características y las herramientas que Moodle puede ofrecer, adicionalmente que se ajuste al contexto Latinoamericano y específicamente a las universidades quienes en la actualidad son las que están sumidas a encontrar nuevas metodologías que mejoren sus entonos virtuales. Es así como Velasco et al. (2020) presentan a la metodología PACIE que permite el mejoramiento de la comunicación y da paso a establecer de manera organizada ambientes pedagógicos en línea, desarrollando el aprendizaje significativo y convirtiendo estas herramientas virtuales principal motivación para la construcción de saberes.

\section{DISCUSIÓN}

Existen estudios que dieron recomendaciones y establecieron benéficos de aplicar Moodle en la enseñanza en el idioma, asimismo, estudios que mostraron los inconvenientes a considerar al momento de aplicar esta plataforma. Colombia conto con el mayor número de artículos escritos refiriéndose al tema tanto en inglés como en español. Sin embargo, estos resultados fueron obtenidos por investigaciones en pequeñas poblaciones.

En la actual era digital se ve indispensable el usar estas plataformas para la enseñanza gracias a todas las funcionalidades que se presentaron con anterioridad. De la misma forma, darle otro enfoque a esta plataforma será de gran ayuda puesto que la mayoría de las producciones científicas reflejaron que al momento de aplicar Moodle como plataforma e-learning en la enseñanza de idioma inglés, los docentes y las instituciones más específicamente las universidades en Latinoamérica acarrean con una gran responsabilidad.

Por esto se enfatizó a la enseñanza en línea como una clave que abre camino a establecer futuros modelos de enseñanza (Zhou et al., 2020). Además, se encuentra que; En los estudios más relevantes mencionados en los tres apartados seguían una misma dirección en donde manifestaban la necesidad de crear ambientes interactivos para que la comunicación sea constante y el uso de esta plataforma se vea de forma positiva para la enseñanza del idioma inglés.

En esta misma línea se descubrió que en los resultados de estos estudios las habilidades comunicativas más desarrolladas por la implementación de Moodle en la enseñanza de inglés en línea tenían como factor común la mejora en la gramática y la escritura.

Por otro lado, es indispensable tomar en cuenta los estudios en donde se relatarán las perspectivas y experiencias de los estudiantes usando la plataforma dado que, en estas investigaciones es donde se vieron los mayores resultados con respecto a los inconvenientes y sugerencias de uso técnico y didáctico por parte de los docentes, si bien es cierto los docentes no tuvieron un amplio conocimiento en el aspecto didáctico, se tiene que considerar la capacitaciones de los mismo para que así mejoren sus habilidades al aplicarlo en la enseñanza de un segundo idioma. El tiempo de adaptación también se presentó como uno de los principales problemas al momento de aplicar Moodle. 
Los resultados de esta investigación concuerdan en que el docente tiene que ser guía, facilitador y en muchos casos flexible en estas plataformas al enseñar un segundo idioma, además de estar preparados para cualquier dificultad o problema que Moodle presente. Se hace una observación con respecto a la integración de Moodle en la enseñanza del idioma ingles y es que en este artículo se rechazó cualquier concepto en el que se descarte el papel principal del educador en el proceso de enseñanza-aprendizaje en la modalidad en línea.

Otro aspecto que se subraya es que a pesar de que el uso de esta plataforma trae grandes beneficios al enseñar el idioma inglés, como suele suceder la mayoría del tiempo en la educación, no todos los docentes podrán considerar esta modalidad en línea como apropiado para la enseñanza de un idioma, a su vez hay estudiantes que prefieren aún la modalidad presencial y no se siente cómodos estudiando en línea. Se infiere que no es solo necesario subir contenido a la plataforma sino también emplearla con un sentido pedagógico. Lo que lleva a replantearse el papel que les otorgan los docentes a estas plataformas en la educación, si bien como un repositorio de información o un espacio activo para la enseñanza y el aprendizaje (Cabero-almenara et al., 2019).

La más grande limitación de este estudio fue que la mayoría de los estudios sus resultados fueron realizados en poblaciones pequeñas y en diferentes contextos ya que existe insuficiente información con respecto al tema de aplicación de Moodle en la enseñanza del idioma inglés, por lo que no se puede llegar a conclusiones tan específicas. Se recomienda también investigar más profundamente los enfoques pedagógicos en esta modalidad en línea a nivel local en el idioma inglés, que ya surge la necesidad de nuevos modelos pedagógicos para llevar a cabo dentro de estos cursos en la plataforma.

En contraste a los beneficios que Moodle ofrece hay que tomar en cuenta que esta plataforma también suele presentar problemas en su integración en los procesos de enseñanza-aprendizaje, que a su vez se convierten en un reto para los docentes, ya sea problemas con el ingreso a la plataforma, envió de tareas o asignaciones, contraseñas o los foros, lo que puede causar confusión, sentimientos de preocupación, frustración, así pues se entiende que la plataforma Moodle tiene limitaciones en cierto punto, adicionalmente se propone actualizar su interfaz y herramienta para mejorar el rendimiento y desarrollo en la enseñanza del idioma inglés.

Otra limitación es que no existe una metodología específicamente dirigida estos modelos de aprendizaje en línea en el idioma inglés que se pudiera nombrar en este artículo, aunque se halla mencionado la metodología PACIE como una alternativa para los entornos virtuales, de está solo se ha rescatado los aspectos positivos de su implementación, por lo que se sugiere a futuras investigaciones enfaticen el empleo de esta metodología dentro del aprendizaje en el idioma ingles a través de Moodle.

\section{REFERENCIAS}

Alcántar, M. del R. C., Torres, C. I., \& Gómez, M. L. M. (2018). Percepción de los estudiantes respecto del uso de las TIC y el aprendizaje del idioma inglés Perception of students regarding the use of ICT and learning English. Apertura (Guadalajara, Jal.), 
I0(2), 50-60. https://doi.org//0.1838I/Ap.v10n2.139I

Ardila, M. E., \& Bedoya, J. R. (2006). La inclusión de la plataforma de aprendizaje en línea MOODLE en un curso de gramática contrastiva español-inglés. Íkala, Revista de $\begin{array}{llll}\text { Lenguaje } y & \text { Cultura, } & \text { II(I7), }\end{array}$ https://www.redalyc.org/articulo.oa?id=2550/255020424007

Aretio, L. G. (2018). Blended learning y la convergencia entre la educación presencial y a distancia Blended learning and the convergence of face-to-face and distance education. Revista Iberoamericana de Educación a Distancia, 2I(I), 09-22. https://doi.org/https://doi.org// 0.5944/ried.2I.I

Arroyave, M. M., Ordóñez, F. E., \& Romero, J. G. (2020). Aproximación al diseño de aulas virtuales universitarias en tiempos de emergencia sanitaria. Espirales Revista Multidisciplinaria de Investigación Científica, 4(34), 67-85. https://doi.org/https://doi.org/I0.3/876/er.v4i34.75 I

Arteaga López, C. (20II). Uso de las TIC para el aprendizaje del inglés en la Universidad Autónoma de Aguascalientes. Apertura, 3(2), 72-79. http://www.udgvirtual.udg.mx/apertura/index.php/apertura/article/view/206/22 I

Barros Bastidas, C., \& Turpo Gebera, O. (2017). La formación en el desarrollo del docente investigador: Una revisión sistemática. Espacios, 38(45). http://www.revistaespacios.com/a I7v38n45/I73845 I I.html

Bonfield, C. A., Salter, M., Longmuir, A., Benson, M., \& Adachi, C. (2020). Transformation or evolution?: Education 4.0 , teaching and learning in the digital age learning in the digital age. Higher Education Pedagogies, 5(I), 223-246. https://doi.org// 0.1080/23752696.2020.1816847

Cabero-almenara, J., Arancibia, M. L., \& Prete, A. Del. (2019). Dominio técnico y didáctico del LMS Moodle en Educación Superior. Más allá de su uso funcional. Journal of New Approaches in Educational Research, 8(I), 27-35. https://doi.org// 0.782I/naer.20I9.1.327

Canals, L., \& Robbins, J. (20I7). An exploratory study of feedback practices for written and oral tasks in an online English course. In K. Borthwick, L. Bradley, \& S. Thouësny (Eds.), CALL in a climate of change: adapting to turbulent global conditions - short papers from EUROCALL 2017 (Pp. 62-66). Research-publishing.net. https://doi.org/https://doi.org/I0.14705/rpnet.2017.eurocall20I7.690

Chávez., R. L. R., Flores, J. G., \& González, R. (2020). Universidad de Guayaquil. In Guía Metodológica Academica Modalidad en Lína de la Universidad de Guayaquil. http://www.ug.edu.ec/secretaria-general-r/normativa/vigente/GUIA METODOLOGICA ACADEMICA MODALIDAD EN LINEA DE LA UNIVERSIDAD DE GUAYAQUIL 2020.pdf 
Cruz, C. P. S., Sedano, C. C., \& Moreno, C. R. (20II). Impacto del uso de la Plataforma Virtual Moodle en el proceso de enseñanza aprendizaje en la Universidad Continental Impact of the usage of the Moodle Virtual Platform in the teaching and learning process in the Continental University. Apuntes de Ciencia \& Sociedad, $0 I(02)$, $120-126$. http://journals.continental.edu.pe/index.php/apuntes/article/view/32

Dávila, A. (20I I). Filosofía Educativa de las aulas virtuales: Caso MOODLE. Compendium, I4(27), 97-105. http://www.redalyc.org/articulo.oa?id=880242I 3006\%0ACómo

Díaz., A. M. C. (20I2). Teaching Foreign Trade in English Through the Modalities Based on Competences and Using Moodle. Issues in Teachers' Professional Development., I4(2), |63-|80. https://www.redalyc.org/articulo.oa?id= |692/|692246350 I |

Domínguez, C. E. G., Romero, J. L. R., Martínez-González, O., \& Piña, I. C. (2019). El uso de las TIC en la Enseñanza del Inglés en las Primarias Públicas. Revista de Estudios y Experiencias En Educación, 18(36), 75-94. https://doi.org/10.21703/rexe.20191836gomez4

Espinoza, J. L. A., Vásquez, R. A. D., \& Yacelga, A. R. L. A. L. S. P. (2019). Moodle: Alternativa de atención a la escolaridad inconclusa en la Unidad Educativa de Imbabura. Dilemas Contemporáneos. Educación, Política y Valores, 6(25), I-2I. https://www.dilemascontemporaneoseducacionpoliticayvalores.com/index.php/dile mas/article/view//442

Figueroa, P. de los Á. G., Gómez, R. M. H., \& Cordero, A. I. E. (2019). Aprendizaje en las redes sociales un enfoque de desarrollo en la comunidad. Revista Tecnológica Ciencia y Educación Edwards Deming, 3(2), 50-66. https://doi.org/https://doi.org// 0.37957/ed.v3i2.35

García, M. E. C., \& López, A. L. (2014). La teoría de las inteligencias múltiples en la enseñanza de las lenguas. Contextos Educativos. Revista de Educación, 17, 79-89. https://doi.org/http://dx.doi.org//0.18172/con.2594

Ghounane, N. (2020). Moodle or Social Networks: What Alternative Refuge Is Appropriate to Algerian EFL Students to Learn during COVID-19 Pandemic. Arab World English Journal, II(3), 2I-4I. https://doi.org/https://dx.doi.org// 0.24093/awej/vol I I no3.2

Gómez, J. E. (2018). Moodle: aplicaciones tecno-comunicativas El caso de la mediación de las TIC en el aprendizaje basado por proyectos de la Universidad Tecnológica de Pereira. Escribanía, 16(I), 223-233. http://scholar.google.com.ar/scholar_url?url=http://revistasum.umanizales.edu.co/o js/index.php/escribania/article/download/2869/3598\&hl=es\&sa=X\&d=45209059/ 3 8319042 I I \&scisig=AAGBfm I xttWH9foVsHtydRjQo7bg9YyF5w\&nossI= I \&oi=sch olaralrt\&hist $=u$ DHZ94sAAAAJ: 136138 
González, J. A. O., \& Medina, S. A. L. (20I2). INTERACTION IN AN EFL READING COMPREHENSION DISTANCE WEB-BASED COURSE. Íkala, Revista de Leguanje y Cultura, I7(I), 45-59. http://www.scielo.org.co/scielo.php?script=sci_arttext\&pid=SOI 23$34322012000100004 \& \operatorname{lng}=e n \& \operatorname{lng}=$

Granados, J., Pilay, L., Ortiz, M., \& Wagner, G. (2019). El docente universitario potenciando el aprendizaje ubicuo mediante el empleo de plataformas de gestión. Espirales Revista Multidisciplinaria de Investigación Científica, 3(24), 160-166. https://doi.org/https://doi.org/I0.31876/er.v3i24.649

Gunduz, N., \& Ozcan, D. (20I7). Implementation of the Moodle System Into EFL Classes Implementación del sistema Moodle en las clases de inglés. Profile Issues in Teachers' Professional Developmen, 19(Suppl. 5I-64. https://doi.org/https://dx.doi.org/I0.15446/profile.vI9n_supI.6857|

Hsiao-fang, C. (20I5). Student's Perceptions of Online Academic English Tutoring. Journal of Pan-Pacific Association of Applied Linguistics, 19(I), 183-194. https://files.eric.ed.gov/fulltext/EJ I085347.pdf

Jakshylykov, J. J., \& Nurmatov, N. A. (2016). Integration challenges of university and information management system ( UIMS ) to Moodle. Integraciâ Obrazovaniâ, 20(2), I58-163. https://doi.org/I0.15507/I99|-9468.083.020.201602.158-163

Joyce, P. (20I8). The Effectiveness of Online and Paper-Based Formative Assessment in the Learning of English as a Second language. PASAA: Journal of Language Teaching and Learning in Thailand, 55(6), 126-146. https://doi.org/10.14456/pasaa.2018.

Knowles, E., \& Kerkman, D. (2007). An Investigation of Students Attitude and Motivation toward Online Learning. InSight: A Journal of Scholarly Teaching, 2, 70-80. https://doi.org/https://doi.org/ 10.46504/02200708kn

Koneru, I. (2017). EXPLORING MOODLE FUNCTIONALITY FOR MANAGING OPEN DISTANCE LEARNING E-ASSESSMENTS. The Turkish Online Journal of Distance I8(4), I29-14I. https://doi.org/https://doi.org// 0.177/8/tojde.340402

Lane, L. M. (20I3). An Open , Online Class to Prepare Faculty to Teach Online. Journal of Educators Online, IO(I), 0I-32. https://doi.org/I0.9743/JEO.20I3.I.I

Luján-García, C., \& Garcia-Sánchez, S. (2015). Moodle as a useful pervasive learning. Calidoscópio, 13(3), 376-387. https://doi.org//0.4013/cld.2015.133.09

Lutfiana, \& Suwartono, T. (2020). Online EFL Teaching and Learning: Advanced Grammar Class and Washback Effect in Test. Technium Social Sciences Journal, I I ( I), 23 - 35. https://doi.org/https://doi.org/I0.47577/tssj.vI Iil.I5I I 
Macancela, E. R. Z., Berrones, W. J. R., Vidal, J. C. P., \& Baque, C. J. S. (2019). Plataformas virtuales y fomento del aprendizaje colaborativo en estudiantes. Sinergias Educativas, 5(I), 350-369. https://doi.org/https://doi.org//0.37954/se.v5il.7I

Ministerio de Educación del Ecuador. (2017). Enfoque de la Agenda Educativa Digital 2017-202I. In Agenda Educativa Digital. https://educacion.gob.ec/wpcontent/uploads/downloads/20 I7/I I/Agenda-Educativa-Digital.pdf.

Mosquera, J. F. V., Fiallos, B. E. A., \& Montenegro, J. E. O. (2019). La educación digital en apoyo a la modalidad. Espirales Revista Multidisciplinaria de Investigación Científica, 3(24), 90-98. https://doi.org/https://doi.org//0.31876/re.v3i24.427

Muhammad. (2020). Promoting Students "Autonomy through Online Learning Media in EFL Class. International Journal of Higher Education, 9(4), 320-33I. https://doi.org//0.5430/ijhe.v9n4p320

Nagi, M., \& Bojiah, J. (2020). Real Classes Vs Online Classes : A Comparative Study on the Chosen Course of HRM Students of Gulf University, Kingdom of Bahrain. International Journal of Emerging Technologies in Learning, I5(I8), 3I-39. https://doi.org/https://doi.org/10.399|/ijet.v15il8.15267

Park, C., \& Kim, D. (2020). Perception of instructor presence and its effects on learning experience in online classes. Journal of Information Technology Education: Research, 19, 475-488. https://doi.org/https://doi.org// 0.28945/46 I I

Peña, Y. P., Pérez, V. E. L., \& Nieves, M. Y. (2020). Actividades para el desarrollo de la competencia comunicativa en inglés a través de la plataforma Moodle. Opuntia Brava, 12(4),

346-357. http://opuntiabrava.ult.edu.cu/index.php/opuntiabrava/article/view// 098

Rey, I. G., García, E. H., \& García, M. R. (2009). Moodle en la enseñanza presencial y mixta del inglés en contextos universitarios. Revista Iberoamericana de Educación a Distancia, I2(I), 169-193. https://doi.org/http://dx.doi.org/I0.5944/ried.I.I2.926

Salmerón-Pérez, H., Rodríguez-Fernández, S., \& Gutiérrez-Braojos, C. (2010). Metodologías que optimizan la comunicación en entornos de aprendizaje virtual. Comunicar Revista Científica de Educomunicación, 17(34), 163 - 171. https://doi.org/I0.3916/C34-2010-03-16

Santamaría, J. S., Antolín, P. S., Javier, F., \& Pardo, R. (20I2). Usos pedagógicos de Moodle en la docencia universitaria desde la perspectiva de los estudiantes. Revista de Estudios y Experiencias En Educación, 60, 15-38. https://doi.org/https://doi.org/ | 0.35362/rie60044 I

Seluakumaran, K., Jusof, F. F., Ismail, R., \& Husain, R. (20I I). Integrating an open-source course management system ( Moodle ) into the teaching of a first-year medical 
physiology course: a case study. Advances in Physiology Education, 35, 369-377. https://doi.org// 0.I I52/advan.00008.20 II.

Shaykina, O. I. (20I5). Blended Learning in English Language Teaching : Open Educational Resources Used for Academic Purposes in Tomsk Polytechnic University. Mediterranean Journal of Social Sciences, 6(3), 255-260. https://doi.org//0.590I/mjss.2015.v6n3s5p255

Srichanyachon, A. N. (2013). Attitudes of Undergraduate Students towards an Online English Class. Turkish Online Journal of Distance Education, 14(2), 225-232. https://dergipark.org.tr/en/pub/tojde/issue// 6896/I 76057

Susanti, Y. (2008). Student Engagement in EFL On-Line Class. Lingual, 10(2), 8-15. https://doi.org/https://doi.org// 0.24843/LJLC.2020.v10.i02.p02

Thamrin, N. S., Suriaman, A., \& Maghfirah. (2019). Students' Perception on the Implementation of Moodle Web-Based in Learning Grammar. IJOTL-TL Indonesian Journal of Language Teaching and Linguistics, 4(I), I-I0. https://doi.org/I0.30957/ijot|tl.v4il.552.Students

Torress-Cajas, M., \& Yépez-Oviedo, D. (2018). Aprendizaje cooperativo y TIC y su impacto en la adquisición del idioma inglés. Revista Mexicana de Investigación Educativa, 23(78), 86 I-882. http://www.redalyc.org/articulo.oa?id=| 4057728009

Vega-carrero, S., Alejandro-pulido, M., \& Ruiz, N. E. (20I7). Teaching English as a Second Language at a University in Colombia That Uses Virtual Environments: A Case Study. Educare Electronic Journal, 2I(3), I-2I. https://doi.org/http://dx.doi.org/I0.15359/ree.21-3.9

Velasco, J. C. C., Gallardo, V. P. S., \& Naranjo, L. M. J. (2020). Mobile-Learning mediated with PACIE methodology for constructivist knowledge. Sophia, Colección de Filosofia de La Educación, 28(I), 139-162. https://doi.org/https://doi.org//0.17163/soph.n28.2020.05

Yafaei, Y. Al, \& Attamimi, R. (2019). Understanding Teachers' Integration of Moodle in EFL Classrooms: A Case Study. English Language Teaching, 12(4), I-6. https://doi.org//0.5539/elt.v|2n4pI

Zawacki-richter, O., Conrad, D., Bozkurt, A., Aydin, C. H., Bedenlier, S., Jung, I., Kalz, M., Kerres, M., Kondakci, Y., Marin, V., Mayrberger, K., Müskens, W., Naidu, S., Qayyum, A., Roberts, J., Sangrà, A., Loglo, F. S., Tryon, P. J. S. van, \& Xiao, J. (2020). Elements of Open Education: An Invitation to Future Elements of Open Education : An Invitation to Future Research. International Review of Research in Open and Distributed Learning, 2I(3), 319-334. https://doi.org/https://doi.org/ /0.19173/irrodl.v2 Ii3.4659 
Zhang, Y., Ghandour, A., \& Shestak, V. (2020). Using Learning Analytics to Predict Students Performance in Moodle LMS. International Journal of Emerging Technologies in Learning, I5(20), https://doi.org/https://doi.org//0.399|/ijet.v15i20.159/5 102-II5.

Zhou, L., Li, F., Wu, S., \& Zhou, M. (2020). "School's out, but Class's on”, the Largest Online Education in the World Today: Taking China's Practical Exploration during the COVID-19 Epidemic Prevention and Control as an Example. Best Evidence of Chinese Education, 4(2), 50I-5I9. https://doi.org/l0.15354/bece.20.ar023. 